\title{
Temporal power reconstruction for an x-ray free-electron laser using convolutional neural networks
}

\author{
X. Ren, A. Edelen $\odot$, A. Lutman, G. Marcus, T. Maxwell, and D. Ratner* \\ SLAC National Accelerator Laboratory, Menlo Park, California 94025, USA
}

(Received 12 December 2019; accepted 30 March 2020; published 9 April 2020)

\begin{abstract}
The free-electron laser process extracts energy from a relativistic electron beam to create high-power, coherent $\mathrm{x}$-ray radiation. The energy loss leaves an imprint of the radiation on the longitudinal energy-time phase-space of the electron beam. At the Linac Coherent Light Source, the X-band transverse deflecting mode cavity measures the longitudinal phase-space, and an x-ray temporal power profile is predicted according to time slices difference between lasing-off and lasing-on measurements. However, the algorithm cannot include physical effects such as slippage in deep saturation and introduces errors from mismatches between the lasing-off and lasing-on measurements. Instead, considering the two-dimensional phase-space as a spatial image, we use a computer vision algorithm to predict the power profile. We simulate thousands of pairs of electron beams and x-ray power profiles to train convolutional neural networks, and test models on both simulated and experimental data. We demonstrate significant improvement compared with the traditional algorithm for a range of conditions.
\end{abstract}

DOI: 10.1103/PhysRevAccelBeams.23.040701

\section{INTRODUCTION}

The Linac Coherent Light Source (LCLS) has been producing femtosecond $\mathrm{x}$-ray free-electron laser (XFEL) pulses from accelerated to $\sim \mathrm{GeV}$ electrons for more than a decade [1]. The ultrashort, intense pulses have facilitated considerable progress in fields as diverse as molecular biology, chemical kinetics analysis, and high energy density science. One of the most important features of XFELs is the ability to resolve dynamics down to the femtosecond or even attosecond scale. The femtosecond scale is critical for studies in protein nanocrystallography damage [2-4], matter under extreme conditions [5], atomic, molecular, and optical physics [6-9], chemical reactions [10], and more. While standard pump-probe experiments are limited in resolution by the minimum duration of the x-ray pulse, knowledge of the temporal power-profile shot-by-shot enables time resolution even shorter than the pulse length [11]; taking an approach of measuring rather than controlling opens the window to both higher time resolution and easier operating conditions [12]. Moreover, pulse length measurements are critical both for development and setup of short pulse modes (see, e.g., [13-16]). However, experimental measurement of the $\mathrm{x}$-ray pulse's temporal properties remains a challenging

*dratner@slac.stanford.edu

Published by the American Physical Society under the terms of the Creative Commons Attribution 4.0 International license. Further distribution of this work must maintain attribution to the author(s) and the published article's title, journal citation, and DOI. problem. Traditional photodetectors cannot resolve femtosecond structure, and time-frequency correlation analysis [17] is not currently feasible in the x-ray regime. Instead, several direct approaches to $\mathrm{x}$-ray measurements in the frequency and time domain have been attempted. In the frequency domain, although spectral analysis and correlation techniques [18-20] extract statistical properties from multiple electron beam shots, these algorithms only return the average pulse duration owing to the loss of phase information. In the time domain, terahertz-field streaking of photoelectrons from a noble gas ionized by the x-ray pulse can characterize a single-shot event with attosecond resolution $[21,22]$. Applying a circularly polarized infrared pulse, the electron spectrometers such as velocity map imaging detectors [23] or angular array of time-of-flight detectors [24] measure the momentum distribution of photoelectrons and can be used to retrieves the power profile by nonlinear fitting [25]. However, streaking methods are only effective for pulses shorter than the streaking laser period, around 10 femtoseconds for the current implementation.

On the other hand, there are indirect methods to retrieve the x-ray pulse profile based on the physics of FEL amplification and the measurement of the longitudinal phase-space downstream of the lasing process. The x-ray pulse derives its energy from the electron beam, so measuring the time-dependent energy loss reveals the $\mathrm{x}$-ray temporal power profile. At LCLS, an X-band transverse deflecting mode cavity (XTCAV) streaks the electron beam in front of the final electron beam dump, providing a measurement of the longitudinal (time-energy) electron beam phase-space [26]. The XTCAV sits downstream from 
the lasing process, but it is also possible to measure the electron beam without lasing ("lasing-off") by suppressing the FEL interaction.

A current method, known as the transverse reconstruction of electron beam and X-ray (TREX) algorithm, reconstructs the temporal power profile by evaluating the product of the measured current profile and the difference between either the energy spread or the mean energy profiles of a lasing-on and a lasing-off shot [27]. However, the TREX algorithm has two shortcomings. First, it is not possible to measure the lasing-on and the lasing-off phase-spaces for a single shot. Instead, an approximate lasing-off phase-space is selected from a pre-recorded set of lasing-off shots by matching the current profile. The shot-to-shot variation in the beams causes mismatches between lasing-on/off examples, leading to erroneous predictions such as predicting negative power. Moreover, owing to the single time slice processing, the algorithm has no capability to deal with the relative slippage of the electrons and x-rays, which limits resolution and assigns power to incorrect times, especially when the XFEL operates in deep saturation.

Rather than using the TREX algorithm, we can instead analyze the entire two-dimensional (2D) XTCAV image. Computer vision (CV) methods can take a raw image as direct input, avoiding the information loss inherent to the TREX analysis. The convolutional neural networks (CNN) have found wide success analyzing images both in industry and in physics [28,29]. In our case, we treat the longitudinal phase-space measurements as 2D images, enabling the application of $\mathrm{CNNs}$ to address the power profile reconstruction problem. We simulate three training data sets consisting of thousands of self-amplified spontaneous emission (SASE) events, each containing the final electron phase-space and the $\mathrm{X}$-ray power profile. The relative root mean square error (RRMSE) of the neural network temporal power predictions are compared with that obtained by the TREX algorithm. We use model uncertainty analysis to evaluate the model robustness and the saliency map to visually connect the input phase-space and output power profiles. Furthermore, transfer learning is used to build a bridge between different FEL modes. Finally, the trained models are applied to experimental XTCAV data.

\section{COMPUTER VISION APPROACH}

Computer vision is an interdisciplinary field that draws from computer science, neuroscience, and analog-digital processing to capture high-level information (classification, localization, or generation) from real-world images or videos, represented as two- to four-dimensional matrices. The deep learning revolution of the last decade has transformed CV through the application of graphics processing units (GPUs), CNN algorithms, and well-labeled, large datasets [30]. These novel CV techniques have found success in a variety of problems, from self-driving cars to facial identification [31]. By treating the electron beam phase-space as a 2D image, we can apply CV algorithms, specifically $\mathrm{CNNs}$, to the power reconstruction problem, i.e., extracting the power profile information from the image of electron beam's longitudinal phase-space.

Two underlying benefits of the CNN are instrumental to the prediction performance. First, the traditional TREX algorithm requires an explicit choice of the lasing-off baseline from a pool of past measurements, leading to errors when the chosen lasing-off phase-space does not perfectly match the lasing-on example. By contrast, the CNN takes only the current lasing-on image as input, and avoids nonphysical negative power predictions sometimes seen with TREX. Second, the slippage of the $\mathrm{x}$-rays relative to the electrons may result in a significant offset between the energy loss imprint on the electron beam and the corresponding x-rays when the FEL reaches deep saturation. This slippage effect is evident in Fig. 2(c), where the energy loss lines angle toward the bottom left. The TREX algorithm works on individual time-slices in the approximation of no significant postsaturation slippage, whereas the CNN model can learn the slippage pattern even in deep saturation. These two advantages are expected to give the CNN improved accuracy over the TREX algorithm.

\section{NEURAL NETWORK TRAINING}

The main challenge to developing an ML solution is creating a well-labeled training dataset that encompasses the range of electron phase-spaces encountered during operation. Ideally, the training data would comprise real experimental data itself so that the training and inference data sets are extracted from the identical probability distribution. However, due to the limitations of the methods for measuring power profiles discussed in Sec. I, the CNN would then be constrained to the performance of the current TREX algorithm. Instead, we design a simulation pipeline as shown in Fig. 1. We first collect archived lasing-off images from the XTCAV and compute the beam current, central energy, and energy spread (standard deviation relative to the central energy) along the time axis. The three functions combined with calibrated time produce realistic electron beam inputs for GENESIS 1.3 [32], a widely-used 3-D FEL simulation code. The undulator "taper" compensates for the electron energy loss with a corresponding reduction in the undulator strength. Measured experimental undulator taper profiles under a lasing-on condition are used to define the taper profile in simulation. GENESIS is then used to generate multiple SASE events for each lasing-off measurement by employing random numerical seeds for loading the electron beam shot-noise. The spent electron beam is further tracked through the dump beamline to the XTCAV longitudinal phase-space measurement using elegant [33], and the resulting macroparticle coordinates are reduced to $2 \mathrm{D}$ longitudinal histograms. These simulations leveraged the SLAC high-performance computing resources as well as 


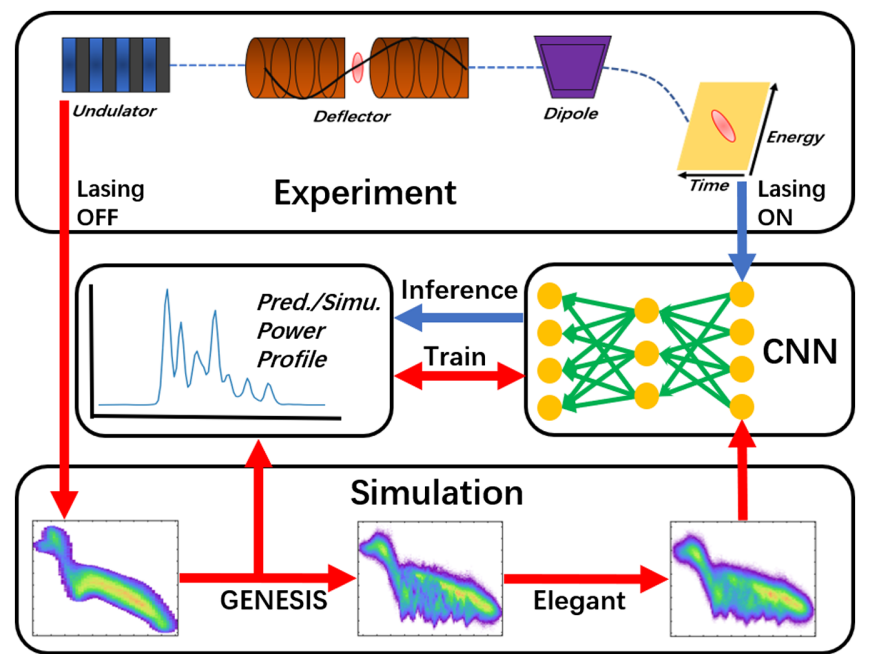

FIG. 1. The power reconstruction problem in the machine learning paradigm. The red flow represents simulation-driven neural network training. The XTCAV measures the lasing-off electron beam, approximating the longitudinal phase-space at the entrance to the undulator. These lasing-off images serve as input to the GENESIS/elegant simulation pipeline, which outputs both the final longitudinal electron beam phase-space and the corresponding x-ray power profile. The simulated electron beam and $\mathrm{x}$-ray power profile pairs then train a convolutional neural network. The blue flow indicates the inference step on new experimental data. We collect the lasing-on phase-space from the XTCAV camera and directly input the plot to the CNN to predict the power profile.

the National Energy Research Scientific Computing Center (NERSC) supercomputer. Finally, the x-ray power profile from GENESIS (smeared to elegant resolution) and 2D electron histograms from elegant serve as the training pairs for the CNN. In addition, the lasing-off phase-space simulations are required for the baseline performance from the TREX algorithm.

Three different soft $\mathrm{x}$-ray experimental setups (beam energy $<5 \mathrm{Gev}$ ) were selected from the previous four years to provide a range of lasing-off records. Using those as input templates, we reproduce in total 5000 shots for the three datasets (named SetShort, SetMedium, and SetLong according to their beam length as shown in Table I). At present, the XTCAV time resolution with maximum voltage at soft $\mathrm{x}$-rays is $\sim 1 \mathrm{fs} \mathrm{rms}$ [26]. For the input images we chose a pixel size of $\sim 0.5 \mathrm{fs} /$ pixel and partitioned all simulated macroparticles as a $2 \mathrm{D}$ histogram. In addition to changing the initial seed on each shot, we introduced randomness to the undulator length so that the simulations include a range of presaturation to postsaturation examples. The change in undulator length captures variation from some unmodeled effects, such as random fluctuations in orbit, match, emittance, etc., that affect the saturation point. For SetLong we simulated more examples, because it has a longer range of undulator lengths, running deep into saturation. Other important configuration parameters are listed in Table I.

The simulated macroparticle histograms and XFEL power profiles serve as input features and output labels of CNN models, respectively. The training data are propagated through the neural network until the loss function converges. To quantify the difference between prediction and true power, we apply the mean square error (MSE) loss function:

$$
\operatorname{MSE} \operatorname{loss}=\sum_{i=1}^{n} \frac{1}{n}\left(p_{i}^{\mathrm{true}}-p_{i}^{\mathrm{pred}}\right)^{2}
$$

where the sum is over all $n$ points in the power profile, $p_{i}$. The neural network is trained using $90 \%$ of each dataset, and we reserve $5 \%$ for validating performance during hyperparameter optimization, and 5\% for testing the model's final performance. We emphasize that the training and testing data sets are simulated from different lasing-off examples; the model has never seen the true lasing-off phase-space for examples in the test set, just as in an actual experiment. More details about datasets, CNN model architecture, and the optimization strategy are described in the Appendix.

\section{RESULTS AND DISCUSSION}

\section{A. Training results and network analysis}

We train separate neural networks for each of the three distinct datasets. To evaluate the performance, we introduce the relative root-MSE error defined as:

$$
\mathrm{RRMSE}=\sqrt{\mathrm{MSE}} / P_{\text {mean }}^{\text {true }},
$$

where $P_{\text {mean }}^{\text {true }}$ is the mean of the ground truth power profile. This unitless quantity eliminates the influence of power magnitude to allow comparisons of performance between different beam configurations and undulator lengths.

TABLE I. Beam parameters and training results for the three datasets. Undulator length (Und. length) randomly chosen within the range given. CNN and TREX Errors are averaged RRMSE on the 5\% of the overall simulations reserved as a test set.

\begin{tabular}{lccccccc}
\hline \hline & Experiment date & Dataset size & Beam length (fs) & X-ray wavelength (nm) & Und. length (m) & CNN error & TREX error \\
\hline SetShort & $02 / 28 / 15$ & 1000 & 27.5 & 1.21 & $30-35$ & 0.101 \\
SetMedium & $11 / 26 / 17$ & 1000 & 73.0 & 2.44 & $40-50$ & 0.209 \\
SetLong & $05 / 06 / 18$ & 3000 & 107.3 & 1.76 & $30-90$ & 0.234 & 0.413 \\
\hline \hline
\end{tabular}



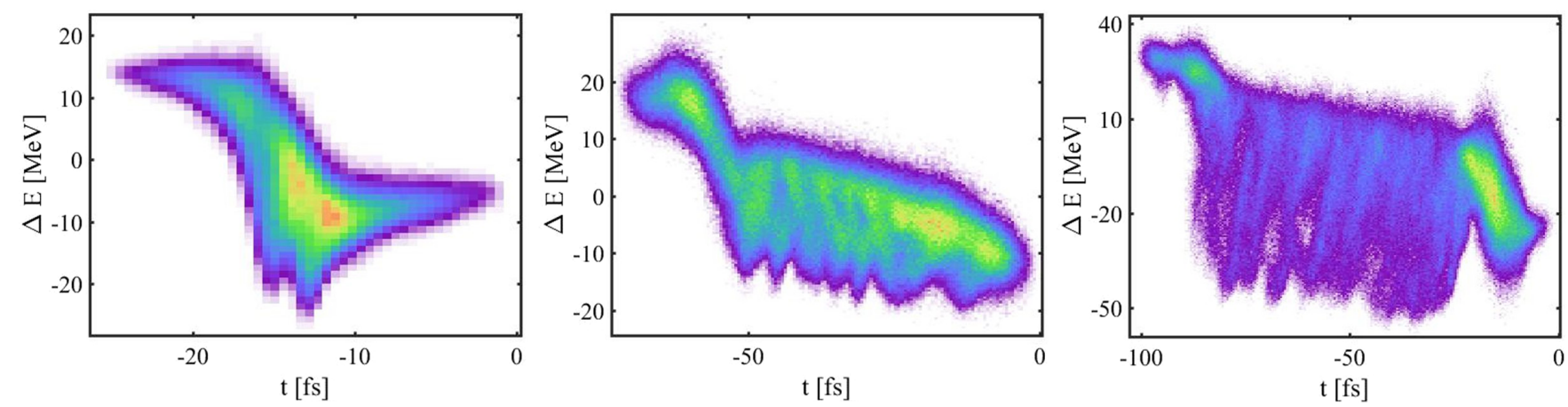

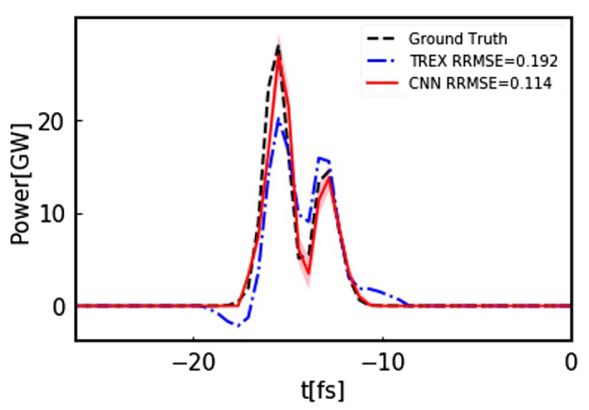

(a) SetShort



(b) SetMedium

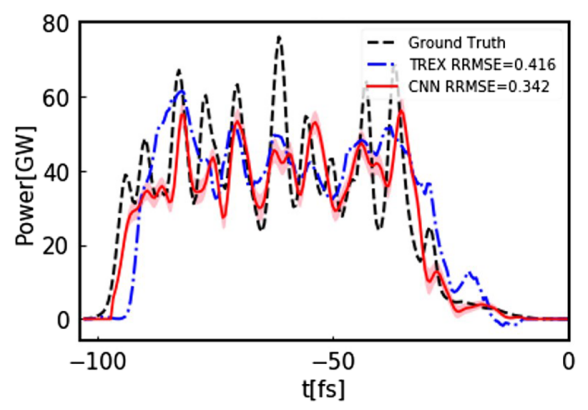

(c) SetLong

FIG. 2. The training simulations and corresponding reconstruction results. The left/center/right three plots are from the Setshort/ medium/long. The upper figures are the simulated output after GENESIS and ELEGANT, and lower plots are the comparisons between the simulated ground truth, TREX algorithm, and CNN prediction. The simulated X-ray power is smeared by convolving with a Gaussian function $(\sigma=0.5 \mathrm{fs})$, while the electron phase-space resolution is determined by the ELEGANT transport ( $\mathrm{rms} \approx 1.3 \mathrm{fs}$ ). The TREX algorithm works with a lasing-off phase-space pool generated from experimental records and evaluates the RRMSE energy spread between lasing-on/off. The error bar of the CNN prediction represents the standard deviation of the network output using the dropout method [34]. The undulator lengths for the three sets are 33/49/90 m respectively, corresponding to approximately saturation for SetShort and SetMedium, and deep saturation for SetLong.

Figure 2 shows examples of lasing-on phase-spaces and corresponding power profiles from the simulation (i.e., ground truth), TREX prediction, and CNN prediction. In all three cases, the CNN prediction has lower RRMSE than that obtained by the TREX algorithm. In SetShort, the TREX algorithm predicts nonphysical positive or even negative power around the beam head and tail region, owing to slight mismatch between the lasing-on phasespace and the lasing-off phase-space pulled from the lasingoff pool. Our lasing-off-free CNN model is able to directly extract lasing spikes from the electron beam and avoid these systematic errors. The intuition is that through scanning many electron phase-spaces, the network learns how to relate the phase-space image to the electron energy loss, i.e., the CNN learns the rule that a spike in energy loss should be associated with a power peak of corresponding size. For SetMedium, in addition to the head/tail mismatch, the traditional algorithm cannot resolve the sharp spikes across the central region; because the spikes overlap in time, the TREX algorithm averages across neighboring spikes. By contrast, our CNN method can find and assign correct lasing patterns spike-by-spike. In deep saturation for SetLong, when the slippage between the x-rays and electrons is significant, the TREX algorithm can no longer correctly place the spikes, while the CNN model captures not only pulse duration but also a majority of spikes, albeit with larger error than earlier in saturation.

Furthermore, we investigate prediction errors as a function of undulator length, which reveals how the two algorithms perform for different saturation degrees. Note that we normalize errors by evaluating RRMSE, eliminating the influence of increasing power in deeper saturation. In Fig. 3, a lower RRMSE $\sim 0.3$ everywhere implies the

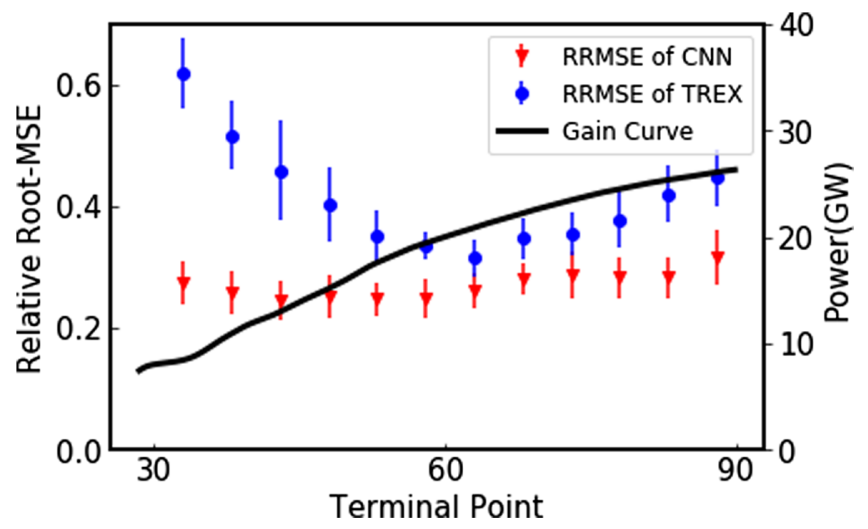

FIG. 3. The SetLong RRMSE for both the CNN (red) and TREX (blue) as a function of undulator length. The gain curve shows the average FEL power vs undulator length. 
CNN model is robust to changes in FEL performance. By contrast, the RRMSE of the TREX algorithm only performs well after saturation $(60 \mathrm{~m})$. In the presaturation region, energy loss of the electron beam is small and difficult to resolve. Those spikes merge and continue to lose energy after saturation, where the TREX algorithm demonstrates the best predictive capability. Deeper in saturation, the TREX error increases again as slippage between the x-ray and electrons grows. By comparison, the $\mathrm{CNN}$ architecture is relatively robust regardless of lasing regime.

The CNN has an additional benefit in its execution time. The TREX has a relatively fast initialization process, but takes approximately $100 \mathrm{~ms}$ seconds per example, depending on the input parameters. By contrast, while training the CNN takes 0.5 hours, once trained forward inference takes $\sim 1 \mathrm{~ms}$. The speed of the CNN makes it possible to do online processing of the XTCAV data.

A danger for models based on deep learning is that successful predictions on the training set do not guarantee correct inference on real data. Good results do not imply the model internally operates according to our expectations, and overfitting is possible due to the substantial number of adjustable parameters in a deep neural network. As an additional check to verify the trained CNN model, we investigate the intermediate layer outputs. Although it is at present not possible to fully explain the behavior of neural networks from a mathematical perspective, there are visualization techniques that contribute to our understanding of how the CNN processes the image. The saliency map, which in our case computes the partial derivative of the layer output with respect to image pixels of phase-space [35], qualitatively evaluates which image pixels are strongly relevant to the output:

$$
S_{i, j}^{I}=\left|\frac{\partial Y^{I}}{\partial X_{i, j}^{I}}\right|
$$

where for each example $Y$ is the scalar layer output (sum over all filters for convolutional layers or all neurons for fully-connected layers), $X_{i, j}$ is one pixel of the phase-space, and $I$ is the specific input phase-space we analyzed. As shown in Fig. 4, the saliency map visually suggests the attention of the CNN: it globally scans the phase-space and
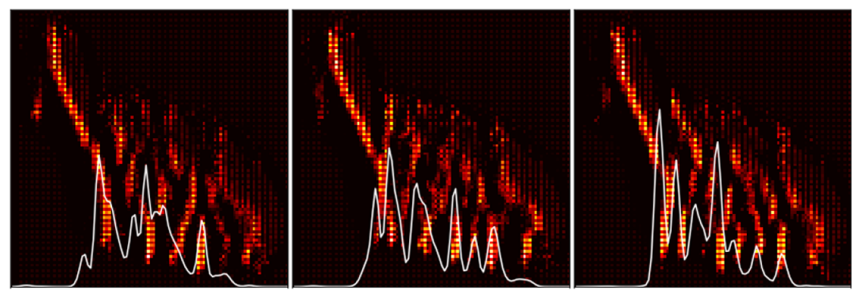

FIG. 4. SetMedium Saliency Map of the first convolutional layer. We use the SetMedium training model for the three example simulations. captures possible lasing strips for subsequent prediction. The focus on the energy loss regions in the saliency map suggests the CNN has learned a physical connection between the electron phase-space and $\mathrm{x}$-ray power profiles without any preknowledge of XFEL physics.

\section{B. Transfer learning}

LCLS frequently changes operating modes, which in turn changes the phase-space, and the three models trained for this paper may not work for new experiments. Rather, users should customize their own model by recording lasing-off XTCAV images during the experiment, and following the procedure of this paper to run simulations and retrain the model.

Simulating a large labeled dataset can be computationally expensive. Transfer learning [36] is an approach to inherit knowledge from pre-trained models to reduce the need for simulation and training. Transfer learning begins with a model pre-trained on an existing, similar data set, and then applies additional rounds of training on a new (and typically smaller) data set. Because the FEL process is consistent across all data sets, we only need to modify existing models with a small number of examples to perform well on a new electron phase-space. For a demonstration of transfer training, we freeze the convolutional layers of the pretrained SetLong model and retrain the model on only 100 samples from SetMedium. The transfer learning model (average RRMSE $=0.315$ ) significantly outperforms a model trained from scratch on the same 100 samples (average RRMSE $=0.436$ and high uncertainty), and is only slightly worse than direct training on the full SetMedium data set (average RRMSE $=0.234$ ). Figure 5 shows the transfer learning results applied to the same example as in Fig. 2(b).

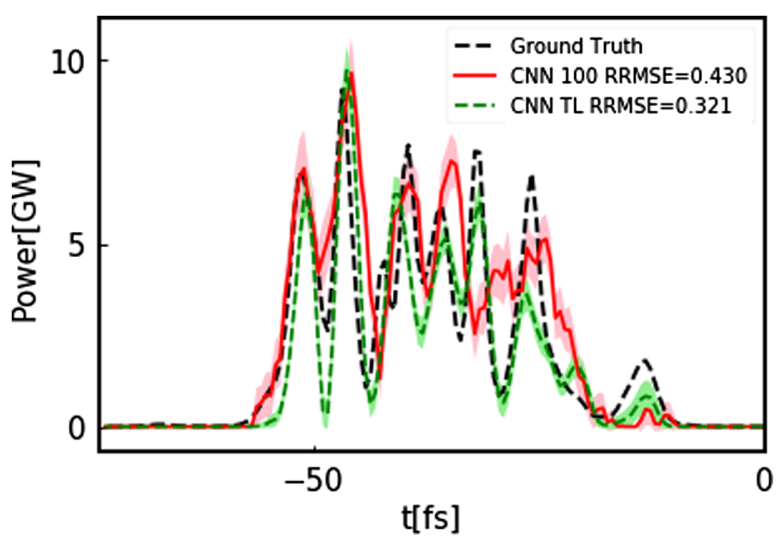

FIG. 5. Transfer learning results of the same SetMedium phasespace in Fig. 2. The red curve is the prediction of a CNN trained on only 100 samples, which shows worse performance than the TREX algorithm due to the small data set. However, using the same 100 samples and the pretrained SetLong CNN to do transfer learning, the prediction (green curve) is nearly as good as the original SetMedium CNN trained on 900 samples (Fig. 2b). 

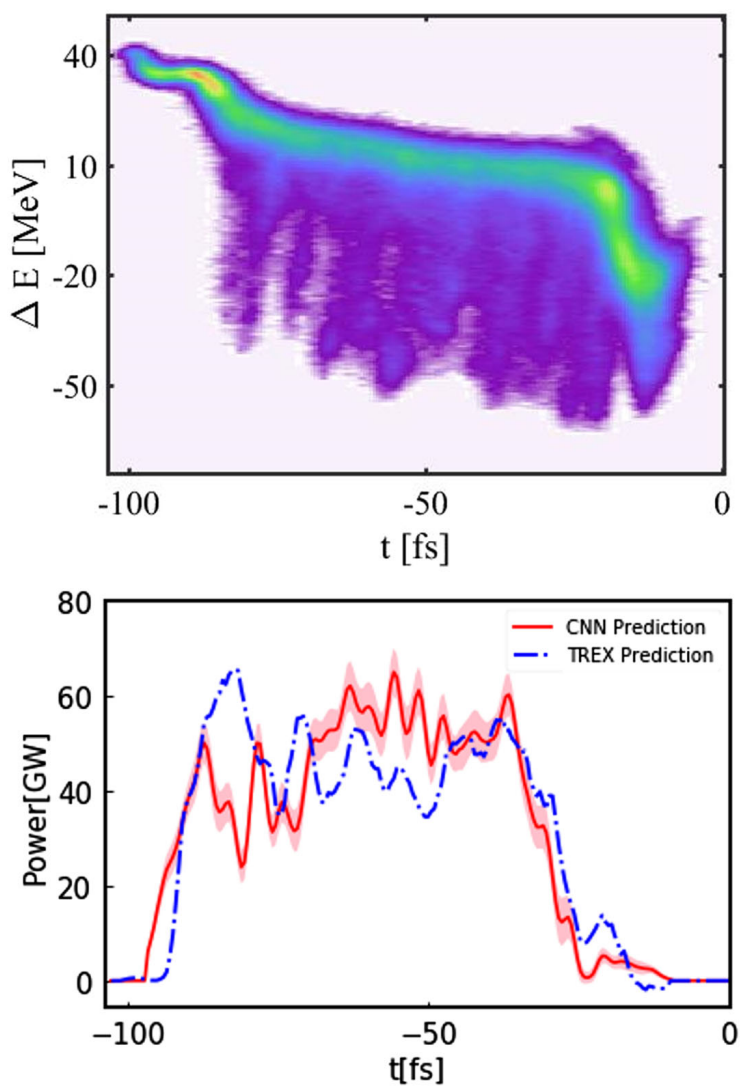

FIG. 6. The experimental XTCAV measurement of a lasing-on phase-space from the SetLong data set, and corresponding predictions from the CNN and TREX algorithm.

\section{Experimental power reconstruction}

Simulation data was used to develop the CNN approach, but ultimately the model needs to be tested on experimental data. To this end, we compare the performance of the CNN with the current TREX algorithm on measured data sets from the LCLS (Fig. 6, a lasing-on record from SetLong). At present, there is no independent method for measuring the power profile, so we can only show a visual comparison between the predictions. As shown in Fig. 6, the two predictions are similar in general contour, but the CNN tends to predict greater variation and the peak locations do not match exactly, perhaps due to slippage effects. Experimental comparison of the two methods will require an independent measurement method, for example user experiments that are sensitive to femtosecond scale fluctuations.

\section{CONCLUSION}

In summary, we have built three XFEL SASE simulation datasets and developed a computer vision-based neural network algorithm for reconstruction of the $\mathrm{x}$-ray power profile from XTCAV data. We demonstrated that the CNN approach provides three advantages over the standard TREX algorithm: (1) it does not require an explicit guess of a lasing-off image to make the prediction and thus avoids lasing-off mismatch errors, (2) it is more robust to slippage effects, and (3) it is $10^{2}$ times faster during inference. The user can directly apply the models if the testing phase-space is close to standard templates or may take advantage of our algorithms and models with new datasets. We also show transfer learning can adapt the current models to new setups with far fewer training examples. As with any other deep neural network algorithms, we can continue improving the model accuracy and range of applicability by learning from new data sets as they become available: one possible improvement would be to train the $\mathrm{CNN}$ on a wider range of beam setups to build a general model that can work on arbitrary configurations. We also note that the output of the $\mathrm{XTCAV}$ analysis can be used as the starting point for additional refinement, for example including spectral information [37]. Finally, we expect this general approach of treating 2D accelerator diagnostics with CNNs can find broad applicability for both users and accelerator physicists.

\section{ACKNOWLEDGMENTS}

We would like to thank Y. Ding, Z. Guo, T. J. Lane, S. Li, T. Raubenheimer, R. Ryne, J. Wu, X. Zhang, Z. Zhang, and G. Zhou for helpful discussions and assistance in simulations. This work was supported by the U.S. Department of Energy under Contract No. DEAC02-76SF00515. Some simulations run at NERSC, a U.S. Department of Energy Office of Science User Facility operated under Contract No. DE-AC02-05CH11231.

\section{APPENDIX: CONVOLUTIONAL NEURAL NETWORK}

The CNN architecture is a sequential model with three convolutional layers and two fully connected layers. The convolutional layers scan the image with 32 nonsquare shape filters (to capture the energy loss characteristic efficiently), and subsequent layers downsample the image and expand along the filter dimension. The convolutional layers use LeakyRelu activation [38] and dropout layers to reduce overfitting. After the convolutional layers, the 3D matrix is flattened to a 1D array for the two fully-connected layers. The output layer predicts the power profile with Relu activation [39]. We use the MSE loss function and the Adam optimizer [40], with a learning rate $5 \times 10^{-5}$ and momentum decay factor $\beta_{1}=0.9, \beta_{2}=0.999$ to train the neural networks with batch size $=50$ until the validation loss function converges.

All XTCAV data are taken from the LCLS Archive. SetShort and SetMedium have 20 different lasing-off samples (two reserved for validation and testing) and we run 50 shots per sample. SetLong has 10 lasing-off samples (one reserved for validation and testing). To increase the diversity of SetLong, 3000 initial phase-spaces are generated by adding a small polynomial variation on the 10 lasing-off beams. 

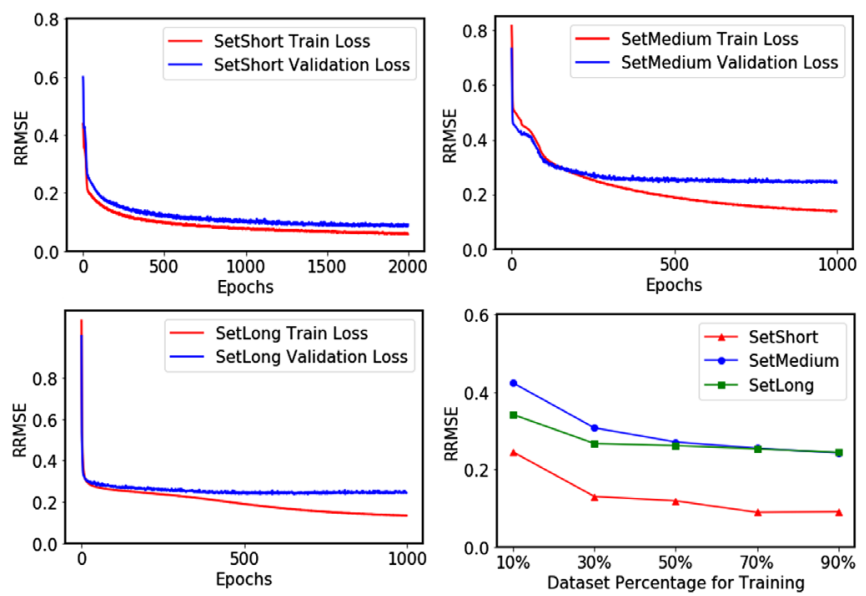

FIG. 7. The three CNN models are trained until the validation loss (blue lines) converges. The bottom right plot gives the validation loss as a function of training dataset size.

Figure 7 shows the evolution of the loss function for the training and validation sets as a function of epoch. The training is terminated after the validation loss converges to prevent overfitting the training dataset. The black curve is the final validation loss when training on different dataset sizes (five marks represent 10\%, 30\%, 50\%, $70 \%$ and $90 \%$ of the whole dataset). The convergence with increasing training sample size suggests that additional samples will not help significantly.

[1] P. Emma et al., First lasing and operation of an ångstromwavelength free-electron laser, Nat. Photonics 4, 641 (2010).

[2] A. Barty et al., Self-terminating diffraction gates femtosecond X-ray nanocrystallography measurements, Nat. Photonics 6, 35 (2012).

[3] K. Nass et al., Indications of radiation damage in ferredoxin microcrystals using high-intensity X-FEL beams, J. Synchrotron Radiat. 22, 225 (2015).

[4] A. Rudenko et al., Femtosecond response of polyatomic molecules to ultra-intense hard X-rays, Nature (London) 546, 129 (2017).

[5] C. A. Stan et al., Liquid explosions induced by X-ray laser pulses, Nat. Phys. 12, 966 (2016).

[6] K. R. Ferguson et al., Transient lattice contraction in the solid-to-plasma transition, Sci. Adv. 2, e1500837 (2016).

[7] I. Inoue et al., Observation of femtosecond X-ray interactions with matter using an X-ray-X-ray pump-probe scheme, Proc. Natl. Acad. Sci. U.S.A. 113, 1492 (2016).

[8] T. Wolf et al., Probing ultrafast $\pi \pi^{*} / n \pi^{*}$ internal conversion in organic chromophores via K-edge resonant absorption, Nat. Commun. 8, 29 (2017).

[9] Y. Pertot et al., Time-resolved x-ray absorption spectroscopy with a water window high-harmonic source, Science 355, 264 (2017).

[10] A. R. Attar, A. Bhattacherjee, C. Pemmaraju, K. Schnorr, K. D. Closser, D. Prendergast, and S. R. Leone,
Femtosecond $\mathrm{x}$-ray spectroscopy of an electrocyclic ringopening reaction, Science 356, 54 (2017).

[11] D. Ratner, J. P. Cryan, T. J. Lane, S. Li, and G. Stupakov, Pump-Probe Ghost Imaging with SASE FELs, Phys. Rev. X 9, 011045 (2019).

[12] T. J. Lane and D. Ratner, What are the advantages of ghost imaging? Multiplexing for x-ray and electron imaging, Opt. Express 28, 5898 (2020).

[13] Y. Ding et al., Generating femtosecond X-ray pulses using an emittance-spoiling foil in free-electron lasers, Appl. Phys. Lett. 107, 191104 (2015).

[14] A. A. Lutman et al., Fresh-slice multicolour X-ray freeelectron lasers, Nat. Photonics 10, 745 (2016).

[15] S. Huang, Y. Ding, Y. Feng, E. Hemsing, Z. Huang, J. Krzywinski, A. A. Lutman, A. Marinelli, T. J. Maxwell, and D. Zhu, Generating Single-Spike Hard X-Ray Pulses with Nonlinear Bunch Compression in Free-Electron Lasers, Phys. Rev. Lett. 119, 154801 (2017).

[16] W. Qin, Y. Ding, A. A. Lutman, and Y.-C. Chao, Matchingbased fresh-slice method for generating two-color X-ray free-electron lasers, Phys. Rev. Accel. Beams 20, 090701 (2017).

[17] G. De Ninno et al., Single-shot spectro-temporal characterization of XUV pulses from a seeded free-electron laser, Nat. Commun. 6, 8075 (2015).

[18] A. Lutman, Y. Ding, Y. Feng, Z. Huang, M. Messerschmidt, J. $\mathrm{Wu}$, and $\mathrm{J}$. Krzywinski, Femtosecond $\mathrm{X}$-ray free electron laser pulse duration measurement from spectral correlation function, Phys. Rev. Accel. Beams 15, 030705 (2012).

[19] R. Mitzner et al., Direct autocorrelation of soft-x-ray free-electron-laser pulses by time-resolved two-photon double ionization of He, Phys. Rev. A 80, 025402 (2009).

[20] Y. Ding et al., Femtosecond X-Ray Pulse Characterization in Free-Electron Lasers Using a Cross-Correlation Technique, Phys. Rev. Lett. 109, 254802 (2012).

[21] U. Frühling et al., Single-shot terahertz-field-driven X-ray streak camera, Nat. Photonics 3, 523 (2009).

[22] I. Grguraš et al., Ultrafast X-ray pulse characterization at free-electron lasers, Nat. Photonics 6, 852 (2012).

[23] S. Li et al., A co-axial velocity map imaging spectrometer for electrons, AIP Adv. 8, 115308 (2018).

[24] N. Hartmann et al., Attosecond time-energy structure of X-ray free-electron laser pulses, Nat. Photonics 12, 215 (2018).

[25] S. Li, Z. Guo, R. N. Coffee, K. Hegazy, Z. Huang, A. Natan, T. Osipov, D. Ray, A. Marinelli, and J. P. Cryan, Characterizing isolated attosecond pulses with angular streaking, Opt. Express 26, 4531 (2018).

[26] Y. Ding, C. Behrens, P. Emma, J. Frisch, Z. Huang, H. Loos, P. Krejcik, and M. Wang, Femtosecond X-ray pulse temporal characterization in free-electron lasers using a transverse deflector, Phys. Rev. Accel. Beams 14, 120701 (2011).

[27] C. Behrens et al., Few-femtosecond time-resolved measurements of X-ray free-electron lasers, Nat. Commun. 5, 3762 (2014).

[28] Y. D. Hezaveh, L. P. Levasseur, and P. J. Marshall, Fast automated analysis of strong gravitational lenses with 
convolutional neural networks, Nature (London) 548, 555 (2017).

[29] L. Dominé and K. Terao, Scalable deep convolutional neural networks for sparse, locally dense liquid argon time projection chamber data, arXiv:1903.05663.

[30] A. Canziani, A. Paszke, and E. Culurciello, An analysis of deep neural network models for practical applications, arXiv:1605.07678.

[31] A. Bhandare, M. Bhide, P. Gokhale, and R. Chandavarkar, Applications of convolutional neural networks, Int. J. Comput. Sci. Inf. Technol. 7, 2206 (2016).

[32] S. Reiche, GENESIS 1.3: a fully 3D time-dependent FEL simulation code, Nucl. Instrum. Methods Phys. Res., Sect. A 429, 243 (1999).

[33] M. Borland, Elegant: A flexible SDDS-compliant code for accelerator simulation, Argonne National Lab., IL (US) Technical Report. LS-287, 2000, https://www.osti.gov/ biblio/761286.

[34] Y. Gal and Z. Ghahramani, Dropout as a bayesian approximation: Representing model uncertainty in deep learning, in Proc. ICML (2016), p. 1050, http://proceedings .mlr.press/v48/gal16.pdf.
[35] K. Simonyan, A. Vedaldi, and A. Zisserman, Deep inside convolutional networks: Visualising image classification models and saliency maps, arXiv:1312.6034.

[36] L. Torrey and J. Shavlik, Transfer learning, in Handbook of research on machine learning applications and trends: algorithms, methods, and techniques (2010), p. 242, http:// ftp.cs.wisc.edu/machine-learning/shavlik-group/torrey .handbook09.pdf.

[37] F. Christie et al., Temporal X-ray reconstruction using temporal and spectral measurements at LCLS, arXiv:1911 .06090 .

[38] A. L. Maas, A. Y. Hannun, and A. Y. Ng, Rectifier nonlinearities improve neural network acoustic models, in Proc. ICML, Vol. 30 (2013), p. 3, https://ai.stanford.edu/ $\sim$ amaas/papers/relu_hybrid_icml2013_final.pdf.

[39] V. Nair and G. E. Hinton, Rectified linear units improve restricted boltzmann machines, in Proc. ICML (2010), p. 807, https://www.cs.toronto.edu/ fritz/absps/reluICML .pdf.

[40] D. P. Kingma and J. Ba, Adam: A method for stochastic optimization, arXiv:1412.6980. 